\title{
Estimation of the probability of daily fluctuations of incidence of COVID-19 according to official data
}

\author{
Andrey Gerasimov ${ }^{\text {Corresp., } 1}$, Elena Galkina ${ }^{1}$, Elena Danilova ${ }^{1}$, Irina Ikonnikova ${ }^{1}$, Tamara Novoselova ${ }^{1}$, Yuriy L Orlov ${ }^{2}$ \\ , Irina Senenycheva ${ }^{1}$ \\ 1 Department of Medical Informatics and Statistics, I.M. Sechenov First Moscow State Medical University, Moscow, Russia \\ 2 Institute of Digital Medicine, I.M. Sechenov First Moscow State Medical University, Moscow, Russia \\ Corresponding Author: Andrey Gerasimov \\ Email address: andr-gerasim@yandex.ru
}

When studying the dynamics of morbidity and mortality, one should not limit ourselves to analyzing general trends. Interesting information can be obtained from the analysis of deviations in morbidity and mortality from the general dynamics. Comparison of the cases of morbidity or death for adjacent time intervals allows us to find out whether the changes in conditions were for short periods of time and whether the cases of morbidity or death were independent. The article consists of two parts: - Study of the probability distribution (CDF) of the difference between two independent observations of the Poisson distribution; - Application of the results to analyze the morbidity and mortality trends by day for the new coronavirus infection. For the distribution function of the module of difference between two independent observations of the Poisson distribution, an analytical expression has been obtained that allows to get an exact solution. A program has been created, whose software can be downloaded at

http://1mgmu.com/nau/DeltaPoisson/DeltaPoisson.zip. An approximate solution that does not require complex calculations has also been obtained, which can be used for an average of more than 20 . If real difference is greater than expected, it may be in the following cases: - morbidity or mortality varies considerably during the day. That could happen, for example, if the registered number of morbidity on Saturday and Sunday is less than on weekdays due to the management model of the health system, - if the cases are not independent, for example, due to the active identification of infected people among those who have come into contact with the patient. If the difference is less than expected, it may be due to external limiting factors, such as a shortage of test systems for making a diagnosis, a limited number of pathologists to determine the cause of death, and so on. In the analysis of the actual data for COVID-19 it was found that for Poland and Russia, excluding Moscow, the difference in the number of cases and deaths is greater than expected, while for Moscow - less than expected. This may be due to the information 
policy - the effort to somehow reassure the Moscow's population, which in the spring of 2020 had a high incidence rate of the new coronavirus infection. 
1 Estimation of the probability of daily fluctuations of incidence of

2

\section{COVID-19 according to official data}

Andrey Gerasimov $^{1}$, Elena Galkina ${ }^{1}$, Elena Danilova ${ }^{1}$, Irina Ikonnikova ${ }^{1}$, Tamara Novoselova ${ }^{1}$, Yuriy Orlov ${ }^{1}$, Irina Senenycheva ${ }^{1}$

${ }^{1}$ I.M. Sechenov First Moscow State Medical University (Sechenov University), 2-4 Bolshaya Pirogovskaya Street, 119991 Moscow, Russia

* Correspondence author

e-mail: andr-gerasim@yansex.ru

Andrey Gerasimov 105043 Izmailovsky boulevard, 32/23, building 1, apt $78+7(905) 550-5084$

\section{Keywords}

COVID-19, random fluctuations of incidence, mathematical methods of morbidity analysis

\section{Abstract}

When studying the dynamics of morbidity and mortality, one should not limit ourselves to analyzing general trends. Interesting information can be obtained from the analysis of deviations in morbidity and mortality from the general dynamics. Comparison of the cases of morbidity or death for adjacent time intervals allows us to find out whether the changes in conditions were for short periods of time and whether the cases of morbidity or death were independent.

The article consists of two parts:

- Study of the probability distribution (CDF) of the difference between two independent observations of the Poisson distribution;

- Application of the results to analyze the morbidity and mortality trends by day for the new coronavirus infection.

For the distribution function of the module of difference between two independent observations of the Poisson distribution, an analytical expression has been obtained that allows to get an exact solution. A program has been created, whose software can be downloaded at http://1mgmu.com/nau/DeltaPoisson/DeltaPoisson.zip. An approximate solution that does not require complex calculations has also been obtained, which can be used for an average of more than 20 .

If real difference is greater than expected, it may be in the following cases: 
- morbidity or mortality varies considerably during the day. That could happen, for example, if the registered number of morbidity on Saturday and Sunday is less than on weekdays due to the management model of the health system,

- if the cases are not independent, for example, due to the active identification of infected people among those who have come into contact with the patient.

If the difference is less than expected, it may be due to external limiting factors, such as a shortage of test systems for making a diagnosis, a limited number of pathologists to determine the cause of death, and so on.

In the analysis of the actual data for COVID-19 it was found that for Poland and Russia, excluding Moscow, the difference in the number of cases and deaths is greater than expected, while for Moscow - less than expected. This may be due to the information policy - the effort to somehow reassure the Moscow's population, which in the spring of 2020 had a high incidence rate of the new coronavirus infection.

\section{Introduction}

The epidemic process of infectious diseases is a random process (Black et al., 2009; Krause et al., 2018; Simões, Telo da Gama \& Nunes 2008).

Even for very large populations, numbering in the millions or more, the average number of infected people per day is small, and even under stable conditions, random fluctuations in the number of infected people gradually accumulate. Its increase is limited to changes in the level of collective immune status, but this process is slow. The magnitude and nature of random fluctuations in the proportion of infected people can be studied using stochastic options of compartment models of the epidemic process, such as SIS, SIR, SIRS, SEIR, and so on (Liu X, Mubayi A, Reinhold D, Zhu L. 2019; Nakamura GM, Martinez AS. 2019). Within these models, it turns out that stochastic effects generate morbidity waves with a period from several years to several decades, depending on the duration of the disease, its infectivity and so on.

However, along with these, there is also a fluctuation in morbidity and mortality, which manifests itself in short periods, including at the level of days. They may be related not only to drastic changes in the epidemic process, but also to changes in the registration conditions (for example, fewer cases are detected on weekends), as well as to the fact that the detection of cases is not necessarily unrelated events. For example, several cases may be detected during a school survey.

There may also be multiple cases detected simultaneously in the family.

Therefore, the analysis of changes in morbidity and mortality over short time periods can reveal the features of the organization of morbidity detection.

An integral part of the analysis of actual morbidity data is to assess the correctness and comparability of official reporting data (Cooper et al., 2009; Isanaka S et al., 2016)

Differences in incidence may be related not only and not so much to differences in disease risk, but to differences in case detection and recording. Therefore, if in the first region the incidence is higher than in the second under similar conditions, it does not mean that doctors perform worse in the first region, the situation is often the opposite one.

Detecting a case and making a diagnosis is not something unambiguous. 
First, for almost all infectious diseases, the proportion of manifest cases is low. Most cases are asymptomatic or unclear symptomatic, and for the most part remain undetected. Some cases are not detected by referrability, but by active detection efforts in foci and among at-risk groups (Abbott et al., 2017; Leung, Trapman \& Britton, 2018).

Second, incidence depends on diagnosis criteria. For example, an infectious disease can be diagnosed both on symptoms and in case of presence of laboratory confirmation. In the latter case, a large part of them goes recorded as "acute respiratory disease" and "intestinal infection of unclarified epidemiology".

The criteria for diagnosis can vary over time and between countries. For example, the decrease in the incidence of tuberculosis in Russia at a rate of about $10 \%$ observed in the last decade is a consequence of the constant change in diagnosis criteria with their fitting to WHO criteria, where only cases with active bacteriodisposition are considered as tuberculosis, while maintaining constant criteria for diagnosing, the incidence of tuberculosis in Russia would continue to increase (Yu WY, Zhang QQ, Xiao Y, Tan WG, Li XD, Lu PX 2019; Герасимов 2018).

Fluctuations in morbidity can also be associated with organisational aspects. For example, in Brazil, the number of COVID-19 cases detected on Saturday and Sunday is about one and a half times lower than on weekdays. In Russia, there are no differences in the incidence of COVID-19 on weekdays and Sundays, but there is a three times difference in the number of recovered due to the fact that on Saturday and Sunday there are no dismisses from hospitals.

Unfortunately, among the factors influencing official morbidity, there is also a desire to show a picture better than it is in reality, hide flaws and errors and "report nicely". This is usually found in a decrease in morbidity and mortality. An example is the legendary statement of the Belarusian leader that "we have no deaths from COVID, we have deaths with COVID".

However, an analysis of official COVID-19 incidence data revealed another phenomenon: an overly stable incidence, in which the number of cases detected per day hardly changes.

Thus, the traditional epidemiological analysis of the morbidity and mortality rates, designed to seek trends, should be supplemented with an analysis of the severity of differences in the number of cases and deaths over the following intervals. This can provide information about the special characteristics of the epidemic process, as well as about the performance of the identification and registration system of cases and death.

\section{Materials and methods}

Let $\mathrm{A}$ be the number of cases detected over a period of time, including per day. Then if the cases are independent and the number of cases is low compared to the overall population, then A is distributed by Poisson (Герасимов 2014). For the Poisson distribution, variance equals mathematical expectation. Therefore, if $\mathrm{x}_{1}, \mathrm{x}_{2}$ are two independent observations of the Poisson distribution with the same average $\lambda$, then $E\left(x_{1}-x_{2}\right)=E\left(x_{1}\right)-E\left(x_{2}\right)=\lambda-\lambda=0$ and $D\left(x_{1}-x_{2}\right)=D\left(x_{1}\right)+D\left(x_{2}\right)=\lambda+\lambda=2 \lambda$. 
117

Besides, for a sufficiently large mathematical expectation, the Poisson distribution is close to the normal distribution (Stuart 2009). Therefore, when increasing the mathematical expectation of the Poisson distribution, the distribution of value $\left(x_{1}-x_{2}\right)$ tends to a normal distribution with a mathematical expectation equal to zero and variance, equal to $2 \lambda$. Consequently, when increasing $\lambda$, the distribution approximates distribution $2 \lambda \chi_{1}^{2}$,

However, the mathematical expectation of the number of sick is unknown to us. If $\mathrm{x}_{1}, \mathrm{x}_{2}$ are two independent observations of the Poisson distribution with mathematical expectation $\lambda$, then $\Delta=\frac{\left(x_{1}-x_{2}\right)^{2}}{x_{1}+x_{2}}$ is not distributed as $\chi_{1}^{2}$, since, first, the Poisson distribution is not exactly the same as normal and secondly, in expression $\frac{\left(x_{1}-x_{2}\right)^{2}}{x_{1}+x_{2}}$ the same values $\mathrm{x}_{1}, \mathrm{x}_{2}$ are used both to estimate variance, and to estimate mathematical expectation.

The value of distribution function $\Delta=\frac{\left(x_{1}-x_{2}\right)^{2}}{x_{1}+x_{2}}$ can be calculated as

$$
F_{\Delta}(x)=\sum_{k \geq 0, n \geq 0, k+n>0, \Delta \leq x} \frac{e^{-k}}{k !} e^{-\lambda} \frac{e^{-n}}{n !} e^{-\lambda}=\sum_{k \geq 0, n \geq 0, k+n>0, \Delta \leq x} \frac{e^{-k-n}}{k ! n !} e^{-2 \lambda}
$$

where $\Delta=\frac{(k-n)^{2}}{k+n}, \mathrm{k}, \mathrm{n}$ are the natural numbers, $F_{\Delta}()$ - distribution function (cumulative density function) of $\Delta$.

Since it requires a fairly large amount of calculations, a program has been created, whose software can be downloaded at http://1mgmu.com/nau/DeltaPoisson/DeltaPoisson.zip.

Figure 1 below shows the calculated distribution function of $\Delta$ and the value $\chi_{1}^{2}$ distribution function. It can be seen that the calculated distribution functions differ very little from the "chi-squared" -distribution even for a small $\lambda$.

Figure 1 Distribution Functions for Value $\Delta$ for $\lambda=3,10,30$ and for $\chi_{1}^{2}$ distribution

Further increase of $\lambda$ does not change the shape of the distribution, it only becomes smoother, close to continuous, the magnitude of the spikes decreases.

It results into a conclusion that to assess the probability of differences in incidence over time intervals, a sufficiently accurate estimate of the average incidence is not required, since the value $\lambda$ for a not very small absolute incidence has little effect on the distribution under study. 
147

148

149

150

151

152

153

154

155

156

157

158

159

160

161

162

163

164

165

166

167

168

169

170

171

172

173

174

175

176

177

178

179

180

\title{
Results
}

Data on the number of people who became ill and died from COVID-19 were taken from publicly available sources: https://covid.observer/ (Data aggregated by the Johns Hopkins CSSE) and https://xn--80aesfpebagmfblc0a.xn--p1ai/ (Official data for the Russian Federation).

We used data for Russia and Poland for the period from July 1 to August 12, since during this period there was no rapid change in incidence (Figure 2).

Figure 2 Number of cases and deaths from COVID-19 for Russia (separately: Moscow and the all other regions) for the period from 1 July to 12 August and Poland for the period from 2 April to 27 May

Analysis of the severity of differences in morbidity and mortality for Moscow are given in Table 1. Table 1 shows that close values are too common for morbidity data for adjacent days. In particular, the values $\Delta$ that should have been present with the probability $\mathrm{F}_{\Delta}<0.1$ were observed in 11 cases out of 42 , while the probability that the binomial distribution with $\mathrm{N}=42$ and $\mathrm{P}=0.1$ takes values of 11 or more is only $0.23 \%$.

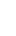

Table 1 The number of COVID-19 cases and deaths by day in Moscow from July 16 to August 12, the magnitude of the differences in incidence $\Delta$ for neighboring days and the probability $\mathrm{F}_{\Delta}$ that such or a lower value may be accidental

There are also valid differences in the $\Delta$ distribution as well.

When analyzing data on Russia (exclusing Moscow) and Poland, we see the following

\begin{abstract}
Table 2 Characteristics of the number of COVID-19 cases and deaths over the periods under review
\end{abstract}

The distributions of observed values, as has already been noted, differ from the expected uniform distribution, but the nature of differences is not the same:

\footnotetext{
Figure 3 Empirical Distributions of Value $\mathrm{F}_{\Delta}$ for Moscow, Russia (excluding Moscow) and Poland
} 
From Figure 3 it follows that for Moscow, both for the number of cases and for the number of deaths, small values of $\Delta$ are more often than expected, while for Russia excluding Moscow and Poland, large values of $\Delta$ are more often than expected.

It follows from Table 2 that:

- For the considered period of relatively stable morbidity, the median of the number of cases of COVID-19 per day in Moscow was 671 cases, the median of incidence differences $\Delta$ is 0.163 , corresponding to $\mathrm{F} \square=0.313$. For the number of deaths from COVID-19 per day we have a median of 14 cases, the median of differences for neighboring days $\Delta$ is 0.154 , which corresponds to $F_{\square}=0.305$. That means, for both the number of cases and the number of deaths, close values for neighboring days were more frequent than expected,

- If one conducts a similar analysis for Russia except Moscow, the median of the number of cases was 5240, the median of the differences for neighboring days $\Delta$ was 0.587 , which corresponds to $F_{\square}=0.555$. For the number of death, the median is 127 , the median $\Delta$ is 2.777 , corresponding to $\mathrm{F}_{\square}=0.904$.

If accepted assumptions correspond to the truth, the value $\mathrm{F}_{\square}$, like any distribution function, must be evenly distributed. When comparing the obtained distributions with uniform using the Kolmogorov-Smirnov criterion, it can be seen (Table 2) that for morbidity in Moscow the difference is true with $\mathrm{p}=0.006$, for Russia excluding Moscow differences are unreliable, $\mathrm{p}=0.126$. At the same time, for mortality in both Moscow and Russia without Moscow, the differences in the actual distribution from the expected are true with $\mathrm{p}<0.001$.

At the same time, for Poland, the differences in both the number of cases and the number of deaths in neighboring days are higher than expected with $\mathrm{p}<0.001$.

\section{Discussion}

When analyzing the infectious morbidity, one of the characteristics is a focality, that is, the degree of grouping of individual cases, which can be a consequence of family hotbeds of disease, foci in organized children's groups, etc. So, if cases are detected not independently, but by $\mathrm{N}$ cases at once, it increases the incidence by $\mathrm{N}$ times, and the variance by $\mathrm{N}^{2}$ times, that is, the ratio between the variance of the number cases and the number of cases can give an estimate about the size a foci.

The assumptions about the independence of individual cases are not entirely accurate, as both the causes of disease and their identification extend the effect not on one person, but on a group of individuals. This is especially pronounced for infectious diseases, as the emergence of a source of the pathogen increases the risk of disease for many contacts, and the detection of one case leads to more active identification among those in contact with him/her. Identifying one case increases the likelihood of detecting other cases, so the variance in the number of cases of disease should be greater than the mathematical expectation.

Also, the variance of morbidity might be increased by changes in the conditions of the epidemic process over time, in which the mathematical expectation of the number of cases and deaths varies over days. 


\section{Conclusion}

\section{References}

Abbott RJ, Pachnio A, Pedroza-Pacheco I, Leese AM, Begum J, Long HM, -Carter DC, Stacey A, Moss PAH, Hislop AD, Borrow P, Rickinson AB, Bell AI. 2017. Asymptomatic primary infection with Epstein-Barr virus: observations on young adult cases. Journal of Virology 91.21:1-49 DOI:10.1128/JVI.00382-17.

Black AJ, McKane AJ, Nunes A, Parisi A. 2009. Stochastic fluctuations in the susceptibleinfective-recovered model with distributed infectious periods. Physical Review E 80.2 DOI: 10.1103/PhysRevE.80.021922.

Cooper GS, Bynum MLK, Somers EC. 2009. Recent insights in the epidemiology of autoimmune diseases: improved prevalence estimates and understanding of clustering of diseases. Journal of Autoimmunity 33.3-4: 197-207. DOI: 10.1016/j.jaut.2009.09.008.

Isanaka S, Boundy EO, Grais RF, Myatt M, Briend A. 2016. Improving Estimates of Numbers of Children With Severe Acute Malnutrition Using Cohort and Survey Data American Journal of Epidemiology. 184.2:861-869 DOI:10.1093/aje/kww129. 
260 Krause AL, Kurowski L, Yawar K, Van Gorder RA. 2018. Stochastic epidemic metapopulation 261 models on networks: SIS dynamics and control strategies. Journal of Theoretical Biology

262 449.14:35-52. DOI: 10.1016/j.jtbi.2018.04.023.

263 Leung KY, Trapman P, Britton T. 2018. Asymptomatic Primary Infection with Epstein-Barr

264 Virus: Observations on Young Adult Cases. Mathematical Biosciences 301:190-198 DOI:

265 10.1016/j.mbs.2018.04.002.

266 Liu X, Mubayi A, Reinhold D, Zhu L. 2019. Approximation methods for analyzing multiscale

267 stochastic vector-borne epidemic models. Mathematical Biosciences 309:42-65. DOI:

268 10.1016/j.mbs.2019.01.003.

269 Nakamura GM, Martinez AS. 2019. Hamiltonian dynamics of the SIS epidemic model with 270 stochastic fluctuations. Sci Rep. 9(1):15841 DOI: 10.1038/s41598-019-52351-x.

271 Simões M, Telo da Gama MM, Nunes A. 2008. Stochastic fluctuations in epidemics on

272 networks. Journal of The Royal Society Interface 5.22: 555-566 DOI: 10.1098/rsif.2007.1206.

273 Stuart A., Ord K. 2009. Kendall's Advanced Theory of Statistics, Distribution Theory 6th

274 Edition. NY, Wiley, 700.

275 Yu WY, Zhang QQ, Xiao Y, Tan WG, Li XD, Lu PX. 2019. Correlation between 18F-FDG PET

276 CT SUV and symptomatic or asymptomatic pulmonary tuberculosis. Journal of X-ray Science

277 and Technology 27.5:899-906 DOI: 10.3233/xst-190543.

278 Герасимов А.Н., Михеева И.В. 2018. Эпидемиологическая ситуация в России - кажущееся

279 благополучие и скрытые угрозы. Тихоокеанский медицинский журнал 73.3:74-78 DOI:

280 10.17238/PmJ1609-1175.2018.3.75-78.

281 Герасимов А.Н. 2014. Определение статистической достоверности различий в

282 заболеваемости. Эпидемиология и Вакцинопрофилактика 78.5:54-60:.

283 https://www.elibrary.ru/download/elibrary_22416303_93752572.pdf 


\section{Table $\mathbf{1}$ (on next page)}

The number of COVID-19 cases and deaths by day in Moscow from July 15 to August 12 , the magnitude of the differences in incidence $\Delta$ for neighboring days and the probability $F_{\Delta}$ that such or a lower value may be accidental 
Table 1

The number of COVID-19 cases and deaths by day in Moscow from July 15 to August 312 , the magnitude of the differences in incidence $\Delta$ for neighboring days and the probability $\mathrm{F}_{\Delta}$ 4 that such or a lower value may be accidental

\begin{tabular}{|c|c|c|c|c|c|c|c|c|}
\hline \multirow[b]{2}{*}{ Date } & \multicolumn{2}{|c|}{$\begin{array}{l}\text { Number of new } \\
\text { cases per day }\end{array}$} & \multicolumn{2}{|c|}{$\Delta$} & \multicolumn{2}{|c|}{$\begin{array}{l}\mathrm{F}_{\Delta}(\mathrm{)}, \text { the exact } \\
\text { solution according } \\
\text { to formula (1) }\end{array}$} & \multicolumn{2}{|c|}{$\begin{array}{l}\mathrm{F}_{\Delta}(), \text { approximate } \\
\text { solution according } \\
\text { to } \chi^{2}{ }_{1} \text { destribution }\end{array}$} \\
\hline & Ill & Dead & Ill & Dead & Ill & Dead & Ill & Dead \\
\hline 16.07 .2020 & 531 & 24 & 8,118 & 0,472 & 0,9956 & 0,5106 & 0,9956 & 0,5078 \\
\hline 17.07 .2020 & 575 & 13 & 1,750 & 3,270 & 0,8143 & 0,9303 & 0,8142 & 0,9295 \\
\hline 18.07.2020 & 578 & 14 & 0,008 & 0,037 & 0,0705 & 0,1569 & 0,0704 & 0,1526 \\
\hline 19.07.2020 & 591 & 14 & 0,145 & 0,000 & 0,2963 & 0,0757 & 0,2962 & 0,0000 \\
\hline 20.07 .2020 & 578 & 15 & 0,145 & 0,034 & 0,2963 & 0,1513 & 0,2962 & 0,1473 \\
\hline 21.07 .2020 & 602 & 17 & 0,488 & 0,125 & 0,5154 & 0,2803 & 0,5152 & 0,2763 \\
\hline 22.07 .2020 & 638 & 19 & 1,045 & 0,111 & 0,6935 & 0,2646 & 0,6934 & 0,2611 \\
\hline 23.07 .2020 & 608 & 14 & 0,722 & 0,758 & 0,6047 & 0,6200 & 0,6046 & 0,6159 \\
\hline 24.07 .2020 & 645 & 11 & 1,093 & 0,360 & 0,7042 & 0,4573 & 0,7041 & 0,4515 \\
\hline 25.07 .2020 & 648 & 14 & 0,007 & 0,360 & 0,0666 & 0,4573 & 0,0665 & 0,4515 \\
\hline 26.07 .2020 & 683 & 9 & 0,920 & 1,087 & 0,6627 & 0,7070 & 0,6626 & 0,7029 \\
\hline 27.07 .2020 & 694 & 13 & 0,088 & 0,727 & 0,2332 & 0,6127 & 0,2331 & 0,6062 \\
\hline 28.07 .2020 & 674 & 10 & 0,292 & 0,391 & 0,4114 & 0,4744 & 0,4113 & 0,4684 \\
\hline 29.07 .2020 & 671 & 13 & 0,007 & 0,391 & 0,0653 & 0,4744 & 0,0652 & 0,4684 \\
\hline 30.07 .2020 & 678 & 12 & 0,036 & 0,040 & 0,1512 & 0,1631 & 0,1512 & 0,1585 \\
\hline 31.07 .2020 & 695 & 14 & 0,210 & 0,154 & 0,3537 & 0,3102 & 0,3536 & 0,3051 \\
\hline 01.08 .2020 & 690 & 13 & 0,018 & 0,037 & 0,1070 & 0,1569 & 0,1069 & 0,1526 \\
\hline 02.08 .2020 & 664 & 12 & 0,499 & 0,040 & 0,5203 & 0,1631 & 0,5202 & 0,1585 \\
\hline 03.08 .2020 & 693 & 13 & 0,620 & 0,040 & 0,5690 & 0,1631 & 0,5689 & 0,1585 \\
\hline 04.08 .2020 & 691 & 12 & 0,003 & 0,040 & 0,0430 & 0,1631 & 0,0429 & 0,1585 \\
\hline 05.08 .2020 & 687 & 11 & 0,012 & 0,043 & 0,0859 & 0,1702 & 0,0858 & 0,1652 \\
\hline 06.08 .2020 & 684 & 13 & 0,007 & 0,167 & 0,0647 & 0,3224 & 0,0646 & 0,3169 \\
\hline 07.08 .2020 & 686 & 12 & 0,003 & 0,040 & 0,0432 & 0,1631 & 0,0431 & 0,1585 \\
\hline 08.08 .2020 & 691 & 14 & 0,018 & 0,154 & 0,1073 & 0,1631 & 0,1072 & 0,3051 \\
\hline 09.08 .2020 & 689 & 12 & 0,003 & 0,154 & 0,0430 & 0,1631 & 0,0429 & 0,3051 \\
\hline 10.08 .2020 & 694 & 13 & 0,018 & 0,040 & 0,1070 & 0,1631 & 0,1070 & 0,1585 \\
\hline 11.08 .2020 & 694 & 14 & 0,000 & 0,037 & 0,0107 & 0,1569 & 0,0000 & 0,1526 \\
\hline 12.08 .2020 & 689 & 12 & 0,018 & 0,154 & 0,1070 & 0,1631 & 0,1070 & 0,3051 \\
\hline
\end{tabular}




\section{Table 2 (on next page)}

Characteristics of the number of COVID-19 cases and deaths over the periods under review 
Table 2

2

Characteristics of the number of COVID-19 cases and deaths over the periods under

3 review

4

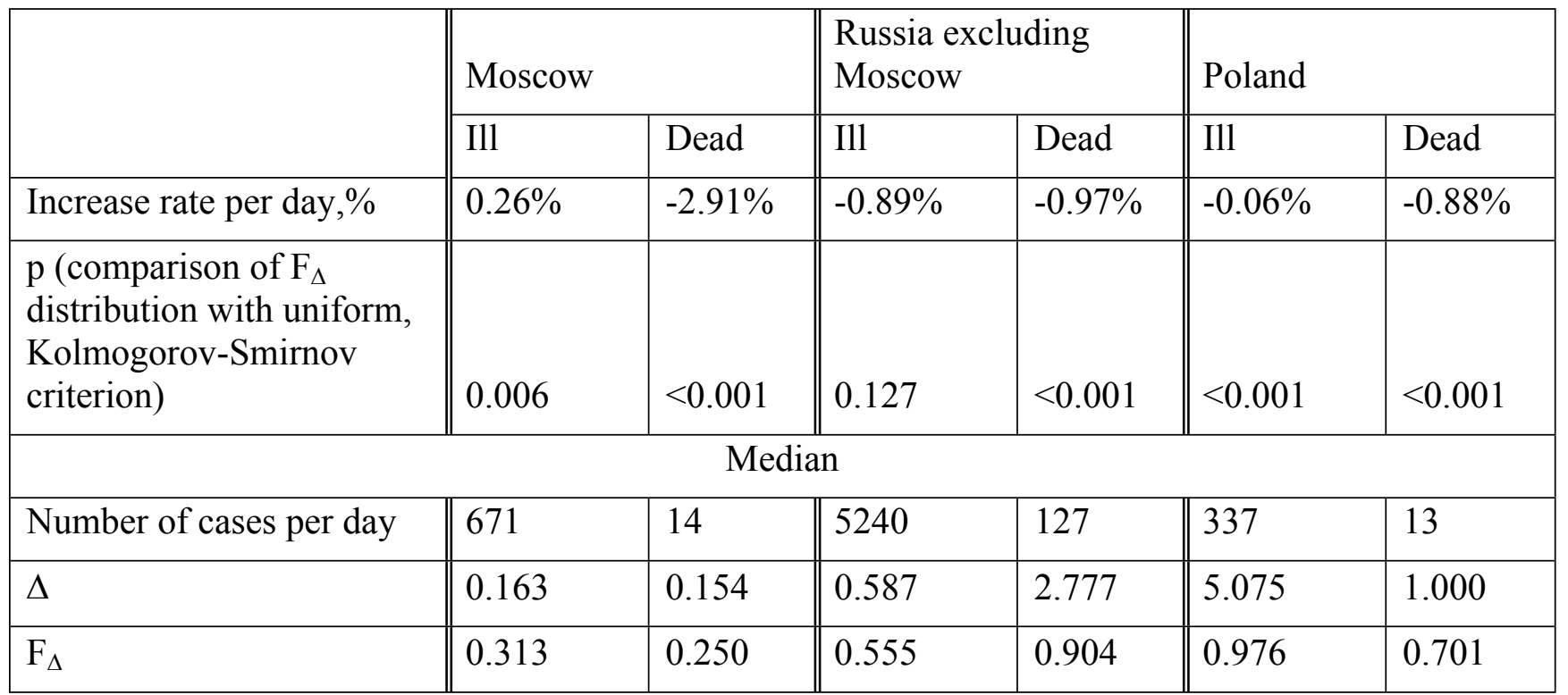

5

6 
Figure 1

Distribution Functions for Value $\Delta$ for $\lambda=3,10,30$ and for $\chi^{2}$ distribution

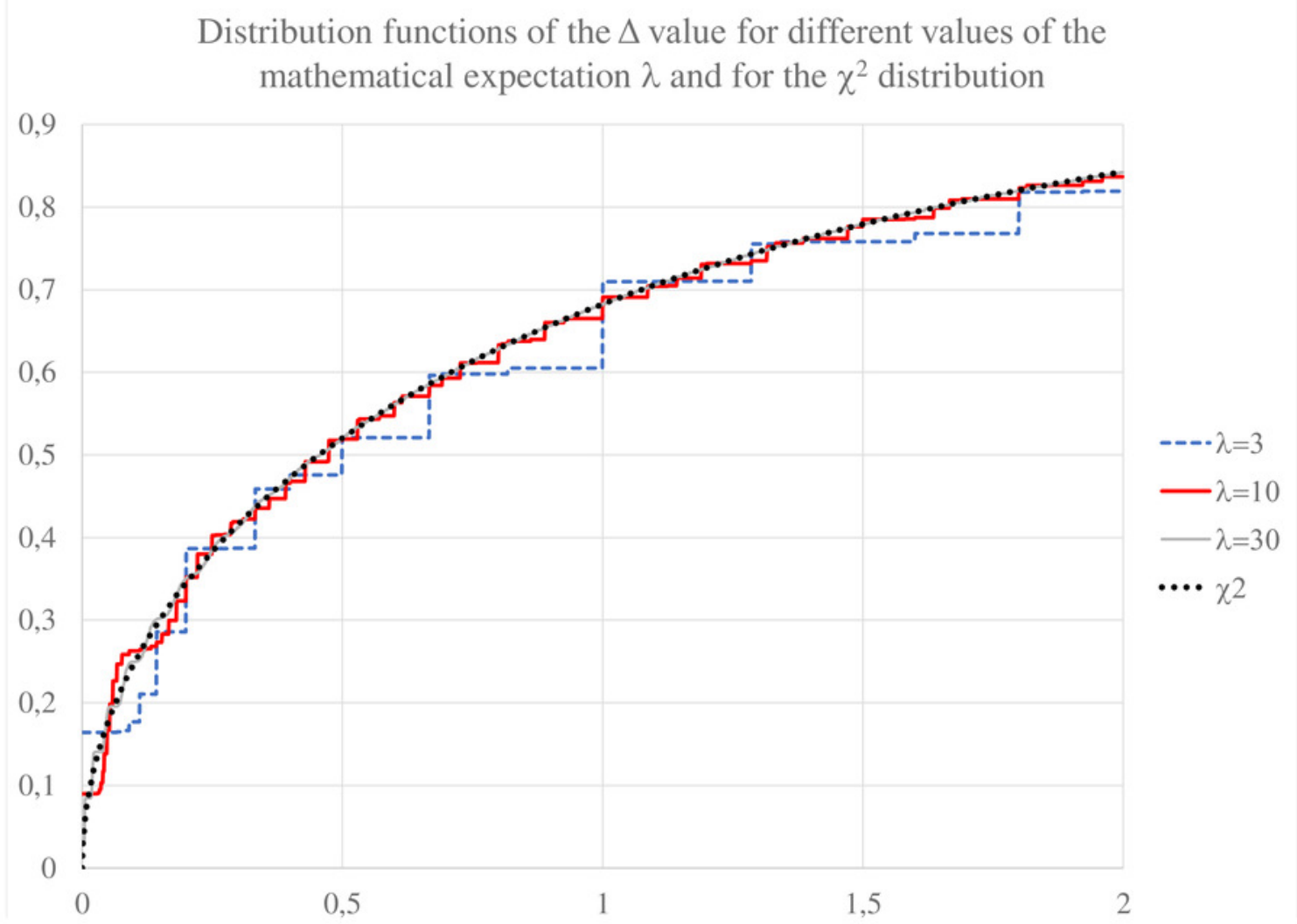


Figure 2

Number of cases and deaths from COVID-19 for Russia (separately: Moscow and the all other regions) for the period from 1 July to 12 August and Poland for the period from 2 April to 27 May

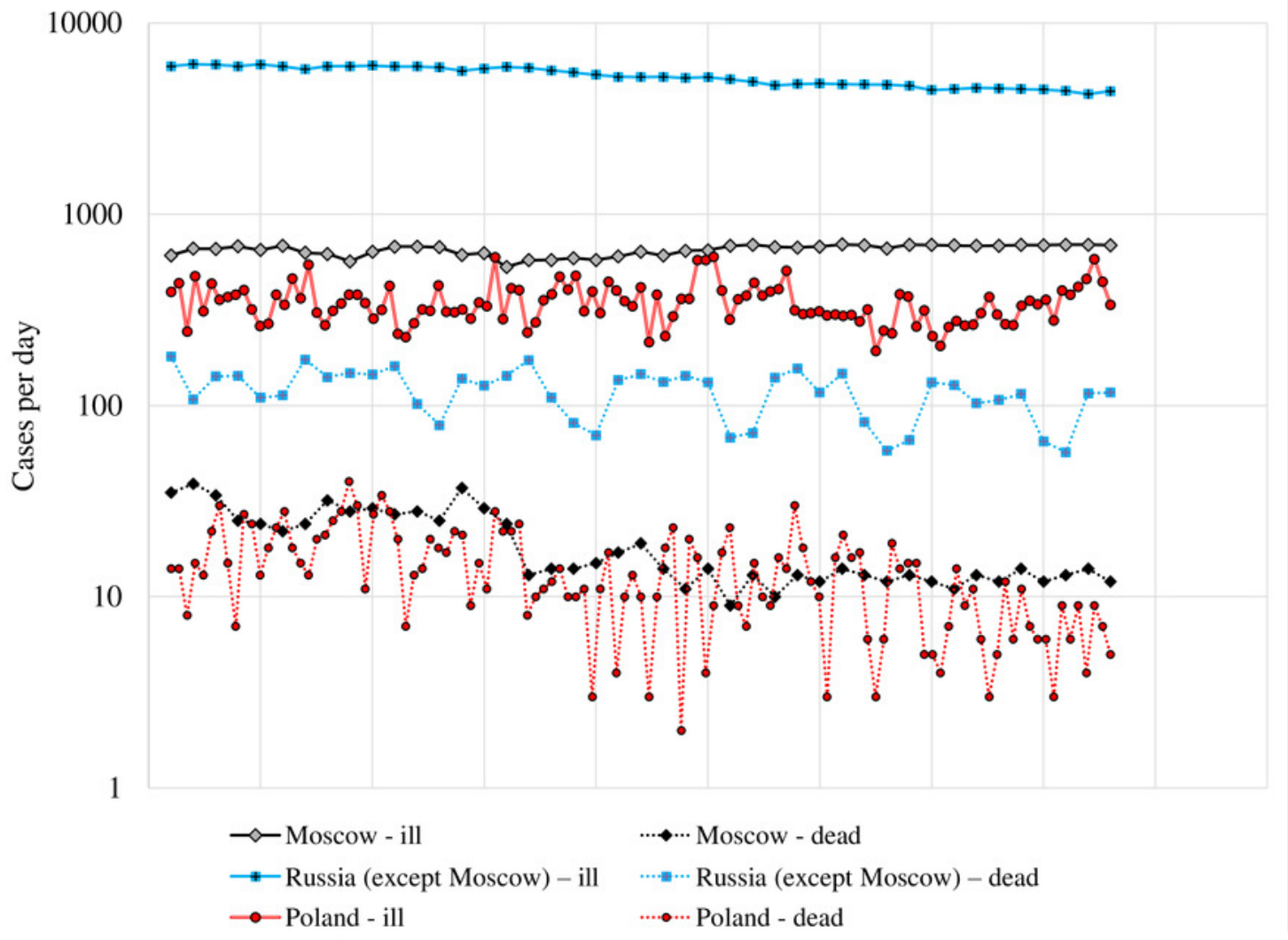


Figure 3

Empirical Distributions of Value $\mathrm{F}_{\Delta}$ for Moscow, Russia (excluding Moscow) and Poland



